\title{
Socio-economic inequalities in stage at diagnosis, and in time intervals on the lung cancer pathway from first symptom to treatment: systematic review and meta-analysis
}

\author{
Lynne F Forrest, ${ }^{1,2}$ Sarah Sowden, ${ }^{1,2}$ Greg Rubin, 2,3 Martin White, ${ }^{1,4}$ Jean Adams ${ }^{4}$
}

\begin{abstract}
- Additional material is published online only. To view please visit the journal online (http://dx.doi.org/10.1136/ thoraxjnl-2016-209013).

IInstitute of Health \& Society, Newcastle University, Newcastle upon Tyne, UK

2Fuse, UKCRC Centre for Translational Research in Public Health, Newcastle University, Newcastle upon Tyne, UK ${ }^{3}$ Wolfson Research Institute, Durham University, Queen's Campus, Stockton on Tees, UK ${ }^{4}$ MRC Epidemiology Unit, University of Cambridge, School of Clinical Medicine, Cambridge Biomedicine Campus, Cambridge, UK
\end{abstract}

Correspondence to Dr Lynne F Forrest, Administrative Data Research Centre Scotland, University of Edinburgh, Edinburgh Bioquarter, 9 Little France Road, Edinburgh, EH16 4UX, UK; Lynne.Forrest@ed.ac.uk

Received 9 June 2016 Revised 25 August 2016 Accepted 1 September 2016 Published Online First 28 September 2016

\section{SLinked}

- http://dx.doi.org/10.1136/ thoraxjnl-2016-209591

CrossMark

To cite: Forrest $L F_{1}$

Sowden S, Rubin G, et al.

Thorax 2017:72:430-436.

\section{ABSTRACT}

Cancer diagnosis at an early stage increases the chance of curative treatment and of survival. It has been suggested that delays on the pathway from first symptom to diagnosis and treatment may be socio-economically patterned, and contribute to socio-economic differences in receipt of treatment and in cancer survival. This review aimed to assess the published evidence for socioeconomic inequalities in stage at diagnosis of lung cancer, and in the length of time spent on the lung cancer pathway. MEDLINE, EMBASE and CINAHL databases were searched to locate cohort studies of adults with a primary diagnosis of lung cancer, where the outcome was stage at diagnosis or the length of time spent within an interval on the care pathway, or a suitable proxy measure, analysed according to a measure of socio-economic position. Meta-analysis was undertaken when there were studies available with suitable data. Of the 461 records screened, 39 papers were included in the review (20 from the UK) and seven in a final meta-analysis for stage at diagnosis. There was no evidence of socio-economic inequalities in late stage at diagnosis in the most, compared with the least, deprived group $(\mathrm{OR}=1.04,95 \% \mathrm{Cl}=0.92$ to 1.19$)$. No socio-economic inequalities in the patient interval or in time from diagnosis to treatment were found. Socioeconomic inequalities in stage at diagnosis are thought to be an important explanatory factor for survival inequalities in cancer. However, socio-economic inequalities in stage at diagnosis were not found in a meta-analysis for lung cancer.

\section{PROSPERO protocol registration number} CRD42014007145.

\section{BACKGROUND}

Patients with cancer who are diagnosed at an early stage are more likely to be suitable for receipt of potentially curative treatment, thus improving their chances of survival. Length of time to diagnosis and treatment has also been associated with cancer survival. ${ }^{1}$ Delays may lead to diagnosis at a later disease stage, resulting in 'potentially avoidable' deaths. $^{2}$ To address this, the National Awareness and Early Diagnosis Initiative scheme was launched in England, in order to encourage early presentation of patients to primary care and to improve general practitioner (GP) cancer recognition and referral. $^{2}$

\section{Key messages}

What is the key question?

- Are there socio-economic inequalities in stage at diagnosis for lung cancer, and in the length of time spent on the lung cancer pathway from symptom onset to treatment?

\section{What is the bottom line?}

- There was no evidence of socio-economic inequalities in late stage at diagnosis in the most, compared with the least, deprived group nor of inequalities in the patient or treatment intervals, and no consistent pattern was observed in diagnostic or referral intervals.

\section{Why read on?}

- Socio-economic inequalities in stage at diagnosis are thought to be an important explanatory factor for survival inequalities in cancer. This is the first study to systematically explore the evidence for inequalities in stage at diagnosis for lung cancer.

Lung cancer is the most common cancer, worldwide. In the USA and the UK, it is the second most incident cancer, ${ }^{3}{ }^{4}$ as well as the most common cause of cancer mortality. ${ }^{4}$ In the UK, fewer than $10 \%$ of those diagnosed with lung cancer survive for 5 years. ${ }^{6}$

A socio-economic gradient for lung cancer survival $^{7}$ exists in the UK, which is not fully accounted for by the socio-economic gradient in incidence. Socio-economic inequalities in receipt of lung cancer treatment have been shown in a recent systematic review and meta-analysis ${ }^{8}$ and there is some evidence that inequalities in treatment contribute to socio-economic inequalities in lung cancer survival. ${ }^{9}{ }^{10}$ It has also been suggested that inequalities in stage at diagnosis and in time to diagnosis and treatment might contribute to socioeconomic differences in cancer survival. ${ }^{2}$ However, there has been no systematic examination of whether there are socio-economic inequalities in stage at diagnosis and time to treatment for lung cancer and where on the pathway from symptom onset to treatment these inequalities might arise. 
This review aimed to investigate whether there are socioeconomic inequalities in stage at diagnosis for lung cancer, and in the duration of intervals (patient, primary care, referral, diagnosis, treatment) on the diagnostic and treatment pathway.

\section{METHODS}

A protocol was developed, registered with PROSPERO ${ }^{11}$ and published. ${ }^{12}$ Systematic methods were used to identify relevant studies, assess study eligibility for inclusion and evaluate study quality. The review is reported according to the Preferred Reporting Items for Systematic Reviews and Meta-Analyses (PRISMA) guidelines ${ }^{13}$ (see online supplementary appendix S1 for PRISMA checklist).

\section{Search strategy}

A search was undertaken to locate all studies published by the initial search date (January 2014), with a title and abstract published in English, examining differences, by socio-economic position (SEP), in stage at diagnosis and in patient, primary care, referral, diagnostic and treatment time intervals (and combinations thereof) on the care pathway for lung cancer. The searches were re-run in February 2016 and any further studies retrieved for inclusion.

One researcher (LFF) developed the search strategy (see online supplementary appendix S2), which was refined with the help of an Information Scientist and used to search the online databases of MEDLINE, EMBASE and CINAHL. The search terms were adapted for the different databases. Additional studies were identified by reviewing the reference lists of all included studies and by using a forward citation search to identify more recent studies that had cited included studies. EndNote X5 software was used to manage the references.

\section{Study eligibility}

The following types of studies were deemed eligible for inclusion: cohort studies of adult participants who had a primary diagnosis of lung cancer (small-cell lung cancer or non-small-cell lung cancer-ICD-10 C33, C34); published in a peer-reviewed journal up to the date that the search was run and where the outcome was:

- Stage at diagnosis or stage at the start of treatment ${ }^{14}$ (primary outcome);

- OR mean or median time within an interval on the pathway from first symptom to treatment (or a suitable proxy measure of this); Or the likelihood (OR or HR with 95\% CIs) of longer or shorter time within an interval on the pathway (secondary outcome);

- And where outcome was analysed by a measure of SEP (an individual or area-based measure of deprivation, poverty, income or education).

\section{Time interval definition}

The following four time points have been identified as important markers on the cancer pathway: date of first symptom onset, date of first presentation to a healthcare professional, date of referral to secondary/specialist care and date of diagnosis. ${ }^{15}$ These and other time points such as date of first secondary (specialist) care investigation/first hospital appointment (FHA), and date of first treatment can be used to define 15 interim time intervals (designated T1-T15) on the pathway from first symptom to diagnosis and treatment. ${ }^{16}$

These time intervals can be categorised as follows: patient interval (time from date of first symptom to date of first presentation; T1); primary care interval (date of first presentation to date of first investigation referral; T6); referral interval (date of GP referral to first specialist appointment or investigation; T10); diagnostic interval (date of secondary care investigation to diagnosis T13) and treatment interval (diagnosis to treatment; $\mathrm{T} 15)^{12}$ and combinations thereof.

The following were included as potential proxy measures of length of time intervals on the pathway; type of referral (urgent vs routine) ${ }^{17}$ emergency presentation, ${ }^{18}$ diagnosis at death and number of prereferral consultations. ${ }^{19}$

\section{Stage definition}

Lung cancer stage can be categorised as I, II, III, IV, assigned using the tumour, node, metastases staging system. ${ }^{20}$ Papers which recorded stage at diagnosis or stage at start of treatment in this way, or as early or late stage, or as local, regional and distant stage, were considered for inclusion in the stage analysis.

\section{Study selection and data extraction}

Studies obtained from the database searches were independently assessed by two researchers (LFF and SS) in three phases: title, abstract and full paper screening, as detailed in the protocol. ${ }^{12}$ Any disagreements at any of the screening stages were resolved by discussion between the two researchers in the first instance. A third reviewer (JA) was available to independently review the title, abstract or full paper, if required.

Data extraction was carried out by LFF using a pro-forma based on previous work. ${ }^{8}$ Data from a random sample of $10 \%$ of included papers were checked by SS, selected using a random number generator.

Insurance status is an important factor relating to access to lung cancer care in the US healthcare system $^{21}$ and so may impact on time intervals on the care pathway. Therefore, as in a previous lung cancer systematic review, ${ }^{8}$ studies were split into three categories: those carried out in a healthcare system free at the point of access (a universal healthcare system (UHCS) similar to the UK); those based on an insurance system (similar to the USA) and those that include a mixture of free care and social insurance-based payment (some European systems).

\section{Study quality}

A study quality checklist, adapted from existing quality tools, ${ }^{8} 15$ was used to divide studies into eight quality categories, with 1 being the lowest and 8 being the highest quality (see online supplementary appendix S3).

Cohort studies reporting only univariable analysis are of lower quality, in terms of their ability to control for confounding. Only studies conducting multivariable analysis (quality scores 3-8) were considered for meta-analysis.

\section{Statistical analysis}

All studies that met the inclusion criteria were included in a narrative synthesis. Studies were analysed in three categories: stage at diagnosis, time interval (with subgroup analyses by interim intervals) and proxy time interval measures.

Studies were considered eligible for inclusion in meta-analysis if: multivariable analysis was conducted (and included control for a minimum of age and sex as confounders); ORs/HRs and $95 \%$ CIs of likelihood of earlier or later stage at diagnosis or longer or shorter time within an interval on the pathway (or a suitable proxy measure of this) in low compared with high SEP were calculated and SEP was not further stratified by another variable. Subgroup analyses by healthcare system category were conducted if two or more comparable studies per system were available. 
Random effects meta-analysis of eligible studies was undertaken using Cochrane Collaboration Review Manager 5.1. Natural logs of the ORs and their SEs were calculated for use in Forest plots. Where a study reported the most deprived class as the comparator, then reverse ORs were calculated prior to meta-analysis. The $\mathrm{I}^{2}$ statistic was used to assess heterogeneity.

Multiple papers using the same or overlapping study data were considered for inclusion. Sensitivity analyses were undertaken to examine the effect of including all potentially eligible studies or only high-quality studies in meta-analyses. However, only data from the better quality or more detailed paper in each overlapping study group were included in the final meta-analyses.

\section{RESULTS}

A total of 39 papers $^{14} 18 \quad 22-58$ met the inclusion criteria (see online supplementary table S1) and were included in the review (PRISMA flow diagram, figure 1). Twenty papers used data from the UK, 10 from the USA, 2 from Canada, 2 from Denmark and 1 each from Sweden, Australia, Italy, Korea and New Zealand. Twenty-nine papers used data from healthcare systems free at point of access and 10 from insurance-based systems. Of the 39 papers, 23 examined stage at diagnosis, 12 examined time intervals ( 3 studies looked at both time intervals and stage at diagnosis) and 8 examined potential proxy measures of delay. Some studies examining stage at diagnosis were suitable for meta-analysis.

\section{Stage at diagnosis}

Of the 23 studies that examined socio-economic inequalities in stage at diagnosis, 16 studies contained data that were unsuitable for meta-analysis (1 reported ORs but examined SEP subdivided by race, 1 calculated univariable ORs and 14 did not calculate ORs -9 presented unadjusted rates (5 with statistical tests) and 5 presented no numbers (see online supplementary table S4). Of these 16 studies, 12 reported or appeared to show no association and 3 reported an association between SEP and stage at diagnosis, but not all in the same direction. One Scottish study $^{28}$ found that rates of early stage cancer were higher in more deprived patients than less deprived. Similarly, a US study $^{54}$ found that college graduates were more likely to be diagnosed with advanced stage at diagnosis compared with those without a college degree, but income was not associated with stage. However, in contrast, a further US study ${ }^{36}$ reported that a higher percentage of more deprived patients were diagnosed with later stage cancer (but no numbers presented).

Eight studies (nine outcomes) conducted multivariable analysis and examined the likelihood (OR) of later (or earlier stage) at diagnosis by a marker of SEP and so were considered for meta-analysis (see online supplementary table S5). Of these, two studies looked at the same population and one study looked at the same outcome using two different measures of SEP (education and income). Seven studies (seven outcomes) with non-overlapping populations were selected for the final meta-analysis.

Overall, there was no evidence of socio-economic inequalities in late stage at diagnosis in the most, compared with the least, deprived groups $(n=7, O R=1.04,95 \% \mathrm{CI}=0.92$ to 1.19) (figure 2). In subgroup analysis by healthcare system, although there was no evidence of differences by SEP in UHCS, there was some suggestion that more deprived patients were likely to present with more advanced stage cancer in non-UHCS, although only two studies were included. Sensitivity analyses including all studies with overlapping populations, using a different measure of SEP, or different combinations of these, also found no association (see online supplementary appendix S6).

\section{Time intervals}

Twelve studies examined inequalities within 8 of the 15 time intervals. No studies were found that examined the intervals
Figure 1 Preferred Reporting Items for Systematic Reviews and Meta-Analyses flow diagram.
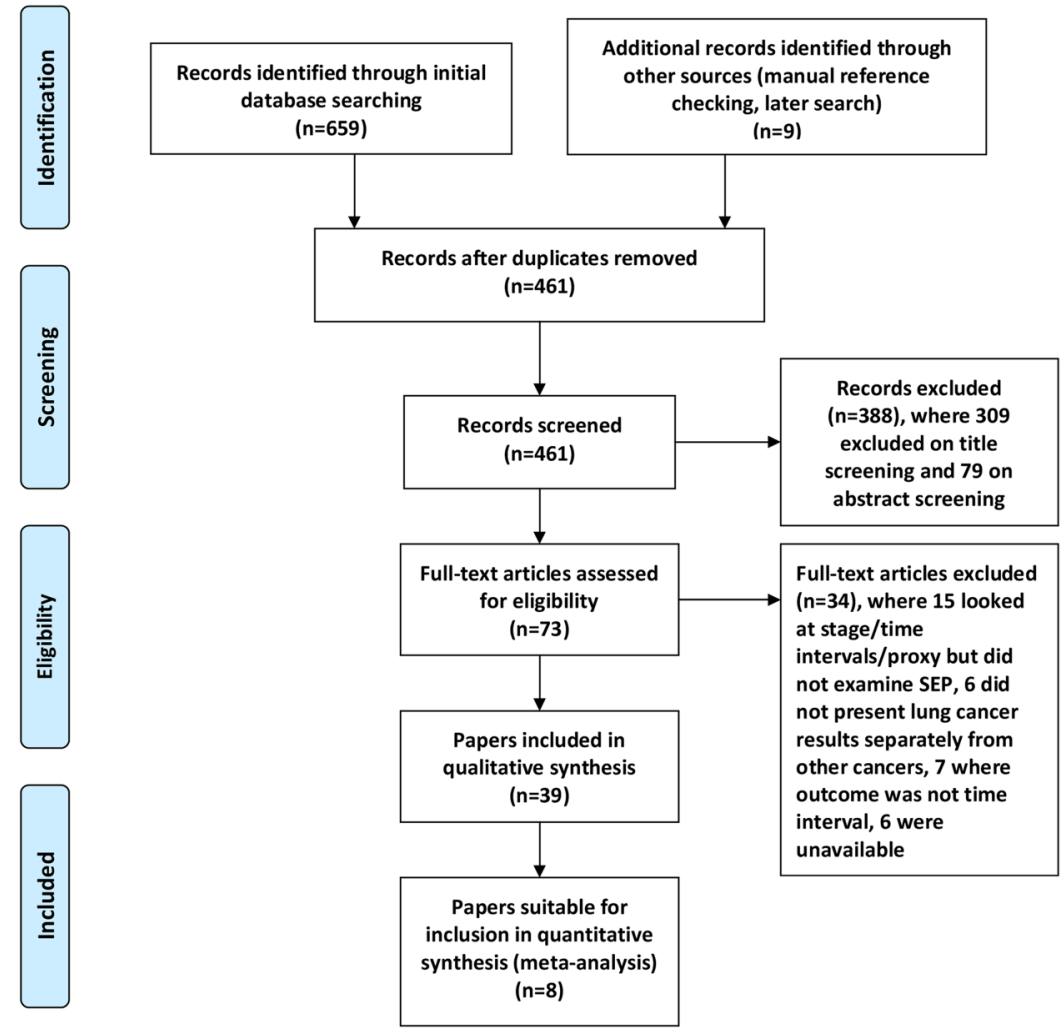


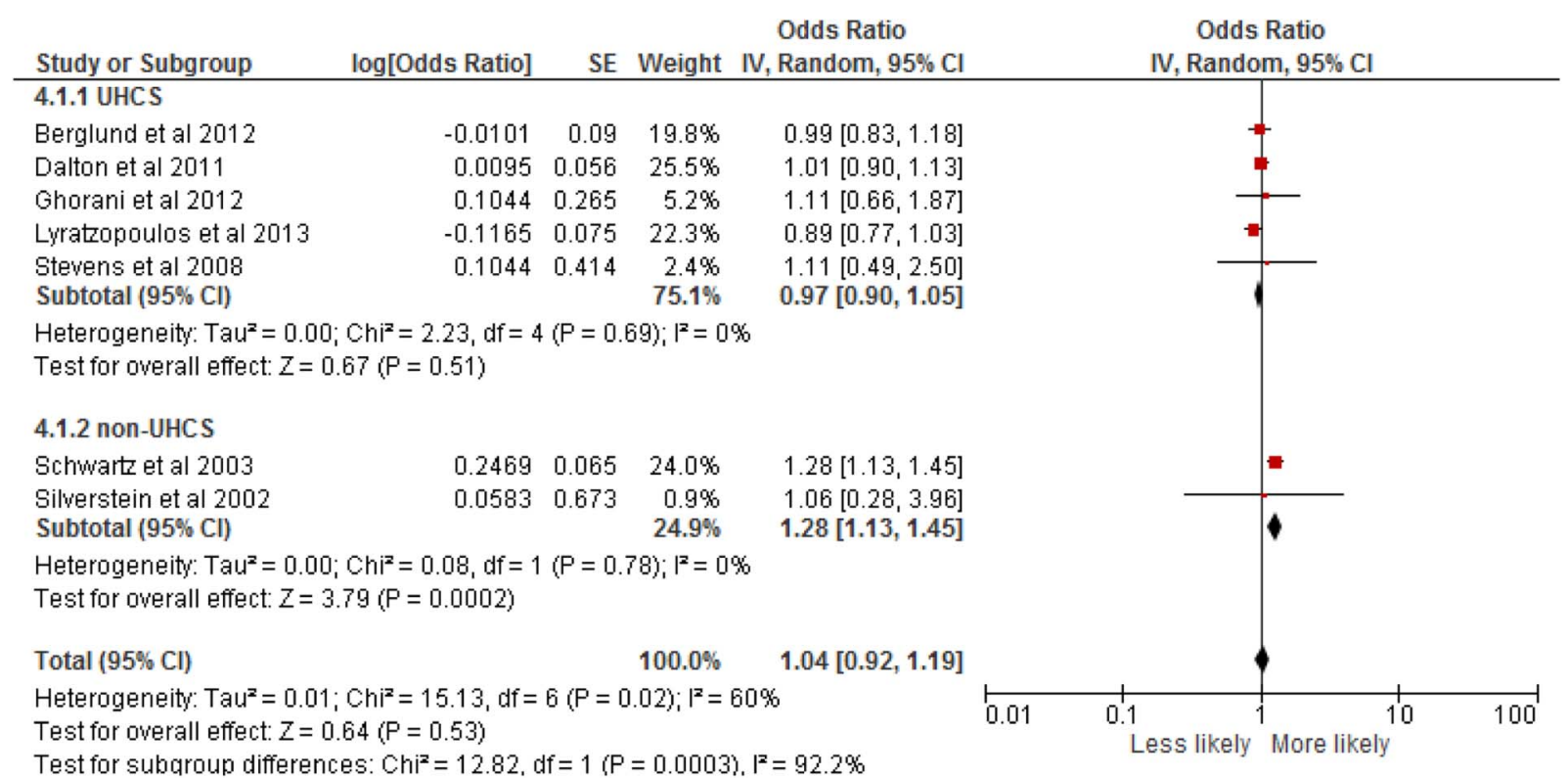

Figure 2 Likelihood of advanced stage at diagnosis in lowest compared with highest socio-economic position group, by universal healthcare system (UHCS) and overall.

from symptom onset to FHA (T3) or to treatment (T5) or from patient presentation to GP referral, FHA, diagnosis or treatment (T6-T9) or FHA to treatment (T14). Summary interval results are shown in figure 3 and detailed results for each interval are presented in online supplementary tables S7-S10.

Two studies examined the time from symptom recognition to presentation (T1 patient interval). SEP was not associated with length of patient interval in any of these (see online supplementary table S7). The intervals from first symptom to GP referral (T2), and to diagnosis (T4), were examined in one study, and again no socio-economic inequalities in time intervals were found (see online supplementary table S7).

Two studies examined the referral interval (T10). One found socio-economic inequalities in time from GP referral to FHA with more deprived patients less likely to have a FHA within 14 days of referral, using cancer registry data. The other used self-reported patient survey data and no association between referral interval and SEP was found (see online supplementary table S8).

Four studies (10 outcomes) examined time from GP referral to diagnosis (T11; see online supplementary table S8). In one study, the more highly educated ( 2 outcomes) were less likely to have delayed diagnosis, but no association was found with income ( 2 outcomes). The other good quality study found no association and the remaining two studies only reported univariable analyses and did not clearly report tests of statistical significance. One of these analysed data from early and late stage patients separately: more deprived early stage patients had longer time from referral to diagnosis than less deprived patients (32 compared with 17 days, although no formal statistical tests were reported). No difference in the time between referral to diagnosis between more and less deprived patients with late stage disease were found.

Two studies examined socio-economic inequalities in time from GP referral to treatment (T12), but none was found (see online supplementary table S8).

Three studies examined time from FHA to diagnosis and none found an association with SEP (T13; see online supplementary table S9). Five studies (eight outcomes) examined socio-economic inequalities in time from diagnosis to treatment (T15), but inequalities in the treatment interval were not found (see online supplementary table S10).

\section{Other measures}

Eight studies looked at the following measures that might be considered as consequences of delay: acute presentation, emergency admission, number of times to consult and diagnosis at death. More deprived patients were more likely to present and to be admitted as an emergency, but socio-economic inequalities were not found in number of times to consult or in diagnosis at death (see online supplementary table S11).

\section{DISCUSSION \\ Principal findings}

This is the first systematic review to explore socio-economic inequalities in stage at diagnosis and in duration of intervals on the diagnostic and treatment pathway of lung cancer. Thirty-nine studies met the inclusion criteria, 23 examined stage at diagnosis (8 of which were suitable for meta-analysis), 12 examined time intervals and 8 examined proxy measures of delay. There was no evidence of socio-economic inequalities in late stage at diagnosis in the most, compared with the least, deprived group overall and in studies from countries with UHCSs. However, there was evidence that more deprived patients were more likely to present as emergencies. There was some suggestion of socio-economic inequalities in stage at diagnosis in studies from countries with non-UHCSs with those in more deprived groups more likely to be diagnosed at late stage than others. No evidence of socio-economic inequalities in the patient or treatment intervals was found, and no consistent pattern was observed in diagnostic or referral intervals. No studies examined the primary care interval.

\section{Interpretation of results and comparison to previous findings}

Surgery with curative intent is only suitable for patients with early stage lung cancer ${ }^{59}$ and if patients with lower SEP are more likely to present later, and with later stage disease, this 


\begin{tabular}{|c|c|c|c|c|c|c|c|c|c|}
\hline & Interval & Symptom onset & $\begin{array}{l}\text { First seen in primary } \\
\text { care }\end{array}$ & $\begin{array}{l}\text { Referral to specialist } \\
\text { care }\end{array}$ & $\begin{array}{l}\text { First seen in } \\
\text { specialist care }\end{array}$ & Diagnosis & Treatment & $\begin{array}{c}\text { N papers (No. of } \\
\text { outcomes) }\end{array}$ & $\begin{array}{l}\mathrm{N} \text { of outcomes } \\
\text { significantly } \\
\text { associated with } \\
\text { length of time in } \\
\text { interval (\%) }\end{array}$ \\
\hline T1 & Patient (P) & & & & & & & $2(4)$ & $0(0)$ \\
\hline T2 & $\mathrm{P}+\mathrm{PC}$ & & & & & & & 1 (1) & $O(0)$ \\
\hline тз & $\mathrm{P}+\mathrm{PC}+\mathrm{R}$ & & & & & & & 0 & - \\
\hline T4 & $P+P C+R+D$ & & & & & & & 1(1) & 0 (0) \\
\hline T5 & $\mathrm{P}+\mathrm{PC}+\mathrm{R}+\mathrm{D}+\mathrm{T}$ & & & & & & & 0 & - \\
\hline T6 & Primary care $(\mathrm{PC})$ & & & & & & & 0 & - \\
\hline T7 & $P C+R$ & & & & & & & 0 & . \\
\hline T8 & $P C+R+D$ & & & & & & & 0 & . \\
\hline т9 & $P C+R+D+T$ & & & & & & & 0 & . \\
\hline T10 & Referral (R) & & & & & & & $2(2)$ & 1 (50) \\
\hline T11 & $R+D$ & & & & & & & $4(10)$ & $3(30)$ \\
\hline T12 & $R+D+T$ & & & & & & & 2 (4) & $O(0)$ \\
\hline T13 & Diagnostic (D) & & & & & & & $3(4)$ & $0(0)$ \\
\hline T14 & $D+T$ & & & & & & & 0 & - \\
\hline T15 & Treatment (T) & & & & & & & $5(8)$ & $0(0)$ \\
\hline
\end{tabular}

Figure 3 Summary of the review findings for each interval.

may help explain known socio-economic inequalities in receipt of lung cancer surgery. ${ }^{8}$ In this review, however, presentation with later stage lung cancer in more deprived patients was not seen and so inequalities in stage at diagnosis cannot explain inequalities in surgery. This concurs with results from our review on socio-economic inequalities in lung cancer treatment, where the association between SEP and receipt of surgery was independent of stage at diagnosis. When receipt of treatment was examined in studies of early stage patients only, low SEP remained associated with reduced likelihood of surgery. ${ }^{8}$

Diagnostic delay has been implicated as a factor that contributes to the poorer survival of the patients with cancer in UK compared with the European average. ${ }^{2}$ However, the impact of delay on lung cancer survival is unclear ${ }^{60}$ and some studies suggest that those who experience shorter system delay may have poorer lung cancer survival as they are clinically 'sicker', with more obvious symptoms that result in urgent referral, diagnosis and treatment. ${ }^{1}$ More deprived patients have been shown to have higher comorbidity. ${ }^{34}$ A recent UK study, which found that patients with lung cancer who were more clinically unwell were referred, diagnosed and treated more quickly, suggested that this 'sicker quicker' effect may act to effectively cancel out socio-economic-related delays that might otherwise result in longer time intervals for more deprived patients. ${ }^{34}$ Very few of the studies included in this current review took account of patient health status when examining time intervals and lack of control for this major confounder may mask any inequalities that are present.

\section{Strengths and weaknesses of the review and of the available evidence}

No previous reviews have systematically assessed socio-economic inequalities in stage at diagnosis and in time intervals on the lung cancer care pathway and few have considered equity in a meta-analysis. Extensive searches were carried out to identify studies in the published literature. However, it is possible that not all relevant studies were obtained.

Although a number of studies examined stage at diagnosis, very few good quality studies examined comparable intervals on the lung cancer diagnostic and treatment pathway, meaning that meta-analysis was only possible for studies examining stage at diagnosis. The included studies reported observational data only. The suitability of meta-analysis for observational studies has been questioned, as it may produce precise but spurious results. ${ }^{61}$ Heterogeneity can be considered high at $>50 \% .^{62}$ Study heterogeneity was taken into account. Separate analyses by healthcare system type were carried out and different effects were found.

There was population overlap between some datasets. We attempted to include only substantially non-overlapping datasets within the final meta-analysis to ensure independence of results. A judgement had to be made as to which was the best quality and most appropriate paper to include, but sensitivity analyses using different inclusion combinations (see online supplementary appendix a) did not change the overall findings nor did including all suitable studies, regardless of population overlap.

A number of different measures of SEP were used in included studies. These likely categorise different people, and different proportions of the population, into the most and least deprived groups, meaning that similar populations are not always being compared across studies. This is a recognised problem in equity reviews. ${ }^{8}$ Most UK studies employ deprivation-index measures of SEP, whereas the US-based studies rely more on income-based measures. However, across all included cases, area-based measures of SEP were common. These may not accurately reflect individual-level circumstances ${ }^{63}$ leading to error, and possible bias. This may mean we have underestimated the strength of the true association between SEP and both stage and intervals. Many included studies used cohort or cancer registry data not specifically collected to study socio-economic inequalities, meaning that the authors of studies included in the review were working with the best available data for secondary analysis. Effort should be made to ensure household, or individual-level, markers of SEP are included in cohort studies and cancer registry datasets to facilitate future analyses.

In the UK only around $20 \%$ of patients are diagnosed at an early stage and so the meta-analysis may be underpowered to detect differences between early and late stage presentation by SEP. 
In order to conduct meta-analysis, it is necessary to compare the odds of advanced stage at diagnosis in the most versus the least deprived groups. This necessarily simplifies what may be a complex relationship across the SEP groups. The ORs used in the meta-analyses were not consistently adjusted for the same covariates. However, we attempted to take this into account in the quality scores and by conducting subgroup sensitivity analyses. Examining only high quality studies did not alter findings nor did other sensitivity analyses. It may be useful to develop clear guidance on what variables should and should not be adjusted for in future analyses to ensure comparability of findings.

Having been unable to find a suitable tool for assessing cohort study quality for a previous review, we created our own. Here, we adapted our previously developed tool ${ }^{8}$ to produce a unique tool that is highly specific to detect bias in the type of study included, considering representativeness of the sample, measure of independent and outcome variables and confounder adjustment. It is similar to a bias assessment checklist tool developed independently in the interim by another review group examining time to diagnosis and treatment in cancer. ${ }^{16}$

Many of the studies included in the narrative review were not of high quality. Many studies used routine data that rely on accurate routine recording of dates and others included selfreport of time intervals, which may suffer from recall bias. There is a possibility of ascertainment bias where sicker patients may be less likely to have stage data recorded. The 'waiting time paradox' where sicker patients progress more quickly through the care pathway as they appear more ill $^{1}$ was not well accounted for and this may mask any true socio-economic inequalities in time intervals. ${ }^{34}$ Lack of consideration of this is a major limitation in the included studies and makes it difficult to draw firm conclusions. Future investigators should routinely include adjustment for patient health status.

Publication bias is a possibility that needs to be considered for any systematic review, as studies reporting null findings may be less likely to be published or, if published, to report numerical outcomes. ${ }^{62}$ However, as the majority of the results reported for this review support the null hypothesis, further unreported null results would not change the findings.

\section{Implications for policy and practice}

No inequalities in the patient interval (from symptom recognition to presentation) or in stage at diagnosis were found in this review. There is no current evidence that more deprived patients wait longer to consult or present with later stage lung cancer. Therefore, there is no indication that campaigns to improve early diagnosis need to be targeted at specific socio-economic groups. Rather these need to be aimed at everyone with symptoms that might potentially indicate lung cancer. However, as more deprived groups have a higher incidence of lung cancer, it may still be prudent to target campaigns at these groups.

Results for the referral and diagnostic intervals were inconclusive, but there was some suggestion of inequalities within these intervals. This could be indicative of clinical bias where, in countries with gatekeeping systems, primary care providers may be more actively referring more affluent patients. Alternatively, patient's ability to navigate through the referral process may vary by SEP. $^{34} 64$

\section{Future research}

Although reducing the time to diagnosis and treatment may have important psychological implications for all patients, ${ }^{16}$ evidence for socio-economic inequalities in time to treatment was not found. However, many studies did not take factors such as cancer stage and health status into account. Better quality studies that attempt to overcome the 'sicker quicker' effect by accounting for health status and examining early stage patients separately are required, to more clearly determine whether inequalities are present.

Socio-economic inequalities in stage at diagnosis have been suggested as an important explanatory factor for survival inequalities in cancer. ${ }^{2}$ However, socio-economic inequalities in stage at diagnosis for lung cancer were not found in UHCSs in the meta-analysis conducted in this review nor was there good evidence for inequalities in time spent on the care pathway. Previous research suggests that socio-economic inequalities in lung cancer treatment rather than in time to treatment might help explain socio-economic inequalities in lung cancer survival. ${ }^{10}$ Further investigation of the patient, tumour and system factors that are important determinants of treatment inequalities is required.

\section{CONCLUSIONS}

There is no clear evidence of socio-economic inequalities in stage at diagnosis or time spent within intervals on the symptom to treatment pathway for lung cancer. However, the quality of evidence available is significantly limited by the failure of most studies to adjust for important potential confounders. Results from this review would suggest that inequalities in time to treatment or in stage at diagnosis are unlikely to account for known socio-economic inequalities in receipt of treatment for, and survival from, lung cancer in UHCSs, but may be present in non-UHCSs.

\section{Twitter Follow Lynne Forrest at @DrLynneForrest}

Contributors LFF: conception and design, data collection and analysis, manuscript writing and final approval of the manuscript. JA, MW and GR: design, critical revision and final approval of the manuscript. SS: design, data collection and analysis, critical revision and final approval of the manuscript.

Funding LFF was funded by the Institute of Health and Society and the Faculty of Medical Sciences, Newcastle University. SS was funded by the National Health Service through Health Education North East. When this work was initiated JA, MW and GR were funded in part as a staff member (JA), director (MW) and senior investigator (GR) of Fuse, the Centre for Translational Research in Public Health (http://www.fuse.ac.uk). Currently, JA and MW are funded by the Centre for Diet and Activity Research (CEDAR). Fuse and CEDAR are UK Clinical Research Collaboration (UKCRC) Public Health Research Centres of Excellence. Funding for Fuse and CEDAR from the British Heart Foundation, Cancer Research UK, Economic and Social Research Council, Medical Research Council, the National Institute for Health Research and Wellcome Trust, under the auspices of the UKCRC, is gratefully acknowledged. The views expressed in this paper do not necessarily represent those of the funders or UKCRC.

Competing interests None declared.

Provenance and peer review Not commissioned; externally peer reviewed.

\section{REFERENCES}

1 Tørring ML, Frydenberg M, Hansen RP, et al. Evidence of increasing mortality with longer diagnostic intervals for five common cancers: a cohort study in primary care. Eur J Cancer 2013;49:2187-98.

2 Richards MA. The national awareness and early diagnosis initiative in England: assembling the evidence. Br J Cancer 2009;101:S1--4.

3 Cancer Research UK. Cancer incidence: UK statistics. London: Cancer Research, 18 Dec 2013. http://info.cancerresearchuk.org/cancerstats/incidence/

4 Howlader NNA, Krapcho M, Neyman N, et al. KA SEER Cancer Statistics Review, 1975-2009 (Vintage 2009 Populations) National Cancer Institute. Bethesda, MD 2011. Based on November 2011 SEER data submission, posted to SEER website 2. http://seer.cancer.gov/csr/1975 2009 pops09/

5 Cancer Research UK. Common cancers-UK mortality statistics. London: Cancer Research, 18 Dec 2013.

6 Allemani C, Weir HK, Carreira H, et al. Global surveillance of cancer survival 1995-2009: analysis of individual data for 25,676,887 patients from 279 population-based registries in 67 countries (CONCORD-2). Lancet 2015;385:977-1010. 
7 Coleman MP, Rachet B, Woods LM, et al. Trends and socioeconomic inequalities in cancer survival in England and Wales up to 2001. Br J Cancer 2004;90:1367-73.

8 Forrest LF, Adams J, Wareham $\mathrm{H}$, et al. Socioeconomic inequalities in lung cancer treatment: systematic review and meta-analysis. PLoS Med 2013;10:e1001376.

9 Forrest $L F$, White $M$, Rubin $G$, et al. The effect of socioeconomic inequalities in receipt of, and time to, treatment on socioeconomic inequalities in lung cancer survival: an observational, data-linkage study. Lancet 2013;382:S37.

10 Forrest LF, White $M$, Rubin $G$, et al. The role of receipt and timeliness of treatment in socioeconomic inequalities in lung cancer survival: population-based, data linkage study. Thorax 2014;70:138-45.

11 Forrest LF, Sowden S, Rubin G, et al. Socio-economic inequalities in patient, primary care, referral, diagnostic and treatment intervals on the lung cancer care pathway: Protocol for a systematic review and meta-analysis. 2014. http://www.crd.york.ac.uk/ PROSPERO/display_record.asp?ID=CRD42014007145

12 Forrest LF, Sowden S, Rubin G, et al. Socio-economic inequalities in patient, primary care, referral, diagnostic, and treatment intervals on the lung cancer care pathway: protocol for a systematic review and meta-analysis. Syst Rev 2014;3:30.

13 Moher D, Liberati A, Tezlaff J, et al., Preferred reporting items for systematic reviews and meta-analyses: the PRISMA statement. PLoS Med 2009;6:e1000097.

14 Lyratzopoulos G, Abel GA, Barbiere JM, et al. Variation in advanced stage at diagnosis of lung and female breast cancer in an English region 2006-2009. Br J Cancer 2012;106:1068-75.

15 Weller D, Vedsted P, Rubin G, et al. The Aarhus statement: improving design and reporting of studies on early cancer diagnosis. Br J Cancer 2012;106:1262-7.

16 Neal RD, Tharmanathan P, France B, et al. Is increased time to diagnosis and treatment in symptomatic cancer associated with poorer outcomes? Systematic review. Br J Cancer 2015;112(Suppl 1):S92-107.

17 Meechan D, Gildea C, Hollingworth L, et al. Variation in use of the 2-week referral pathway for suspected cancer: a cross-sectional analysis. Br J Gen Pract 2012;62: e590-e7.

18 McPhail S, Elliss-Brookes L, Shelton J, et al. Emergency presentation of cancer and short-term mortality. Br J Cancer 2013;109:2027-34.

19 Lyratzopoulos G, Neal RD, Barbiere JM, et al. Variation in number of general practitioner consultations before hospital referral for cancer: findings from the 2010 National Cancer Patient Experience Survey in England. Lancet Oncol 2012;13:353-65

20 Sobin $\mathrm{LH}$, Wittekind $\mathrm{CH}$. TNM classification of malignant tumors. 5th edn. New York: John Wiley \& Sons, 1997.

21 Slatore CG, Au DH, Gould MK, on behalf of the American Thoracic Society Disparities in Healthcare G. An official American Thoracic Society Systematic Review: insurance status and disparities in lung cancer practices and outcomes. Am J Respir Crit Care Med 2010;182:1195-205.

22 Abel GA, Shelton J, Johnson S, et al. Cancer-specific variation in emergency presentation by sex, age and deprivation across 27 common and rarer cancers. $\mathrm{Br}$ J Cancer 2015;112:S129-36.

23 Beckett $\mathrm{P}$, Stanley $\mathrm{R}$, Woolhouse I, et al. S92 Risk factors and outcome for emergency presentation in lung cancer patients [abstract]. Thorax 2012;67(Supp 2): A45-A46. doi:10.1136/thoraxjn-2012-202678.098

24 Beckett P, Tata LJ, Hubbard RB. Risk factors and survival outcome for non-elective referral in non-small cell lung cancer patients-analysis based on The National Lung Cancer Audit. Lung Cancer 2014;83:396-400.

25 Berglund A, Holmberg L, Tishelman C, et al. Social inequalities in non-small cell lung cancer management and survival: a population-based study in central Sweden. Thorax 2010;65:327-33.

26 Berglund $A$, Lambe $M$, Lüchtenborg $M$, et al. Social differences in lung cancer management and survival in South East England: a cohort study. BMJ Open 2012;2:pii: e001048.

27 Booth CM, Li G, Zhang-Salomons J, et al. The impact of socioeconomic status on stage of cancer at diagnosis and survival: a population-based study in Ontario, Canada. Cancer 2010;116:4160-7.

28 Brewster DH, Thomson CS, Hole DJ, et al. Relation between socioeconomic status and tumour stage in patients with breast, colorectal, ovarian, and lung cancer: results from four national, population based studies. $B M J$ 2001;322:830-1.

29 Campbell NC, Elliott AM, Sharp L, et al. Impact of deprivation and rural residence on treatment of colorectal and lung cancer. Br J Cancer 2002;87:585-90.

30 Cheyne L, Taylor A, Milton R, et al. Social deprivation does not affect lung cancer stage at presentation or disease outcome. Lung Cancer 2013;81:247-51.

31 Dalton SO, Frederiksen BL, Jacobsen E, et al. Socioeconomic position, stage of lung cancer and time between referral and diagnosis in Denmark, 2001-2008. Br J Cancer 2011;105:1042-8.

32 Deepak J, Gardner L, Mao L, et al. Socioeconomic disparities and the relationship between COPD and lung cancer. CHEST 2012;142(4_MeetingAbstracts):620A-A

33 Forrest LF. Intervention-generated inequalities in lung cancer care. Newcastle upon Tyne: Newcastle University, 2013

34 Forrest LF, Adams J, White $M$, et al. Factors associated with timeliness of post-primary care referral, diagnosis and treatment for lung cancer: population-based, data-linkage study. Br J Cancer 2014;111:1843-51.
35 Ghorani E, O'Leary B, Asif K, et al. P168 Factors associated with advanced stage lung cancer at diagnosis-a retrospective cohort study [abstract]. Thorax 2012;67 (Supp 2):A135-A136.

36 Gong G, Belasco E, Hargrave KA, et al. Determinants of delayed detection of cancers in Texas counties in the United States of America. Int J Equity Health 2012;11:29.

37 Haas JS, Earle CC, Orav JE, et al. Racial segregation and disparities in cancer stage for seniors. J Gen Intern Med 2008:23:699-705.

38 Halpern MT, Holden DJ. Disparities in timeliness of care for U.S. Medicare patients diagnosed with cancer. Current Oncol 2012;19:e404-13.

39 Hui AC, Vinod SK, Jalaludin BB, et al. Socio-economic status and patterns of care in lung cancer. Aust N Z J Public Health 2005;29:372-7.

40 Johnson AM, Hines RB, Johnson lii JA, et al. Treatment and survival disparities in lung cancer: the effect of social environment and place of residence. Lung Cancer 2014;83:401-7.

41 Jones AP, Haynes R, Sauerzapf V, et al. Geographical access to healthcare in Northern England and post-mortem diagnosis of cancer. J Public Health (Oxf) 2010;32:532-7.

42 Lyratzopoulos $\mathrm{G}$, Abel GA, Brown $\mathrm{CH}$, et al. Socio-demographic inequalities in stage of cancer diagnosis: evidence from patients with female breast, lung, colon, rectal, prostate, renal, bladder, melanoma, ovarian and endometrial cancer. Ann Oncol 2013:24:843-50.

43 Mor V, Masterson-Allen S, Goldberg R, et al. Pre-diagnostic symptom recognition and help seeking among cancer patients. J Community Health 1990;15:253-66.

44 Neal RD, Allgar VL. Sociodemographic factors and delays in the diagnosis of six cancers: analysis of data from the "National Survey of NHS Patients: Cancer". $\mathrm{Br}$ J Cancer 2005;92:1971-5.

45 Niu X, Pawlish KS, Roche LM. Cancer survival disparities by race/ethnicity and socioeconomic status in New Jersey. J Health Care Poor Underserved 2010;21:144-60

46 Pagano E, Filippini C, Di Cuonzo D, et al. Factors affecting pattern of care and survival in a population-based cohort of non-small-cell lung cancer incident cases. Cancer Epidemiol 2010:34:483-9.

47 Pollock AM, Vickers N. Deprivation and emergency admissions for cancers of colorectum, lung, and breast in south east England: ecological study. BMJ 1998;317:245-52

48 Raine R, Wong W, Scholes $\mathrm{S}$, et al. Social variations in access to hospital care for patients with colorectal, breast, and lung cancer between 1999 and 2006: retrospective analysis of hospital episode statistics. BMJ 2010;340:b5479.

49 Saint-Jacques N, Rayson D, Al-Fayea T, et al. Waiting times in early-stage non-small cell lung cancer (NSCLC). J Thorac Oncol 2008;3:865-70

50 Schwartz KL, Crossley-May H, Vigneau FD, et al. Race, socioeconomic status and stage at diagnosis for five common malignancies. Cancer Causes Control 2003:14:761-6.

51 Shin D, Cho J, Kim S, et al. Delay to curative surgery greater than 12 weeks is associated with increased mortality in patients with colorectal and breast cancer but not lung or thyroid cancer. Ann Surg Oncol 2013;20:2468-76.

52 Silverstein MD, Nietert PJ, Ye $\mathrm{X}$, et al. Access to care and stage at diagnosis for patients with lung cancer and esophageal cancer: analysis of the Savannah River Region Information System cancer registry data. South Med J 2002:95:900-8.

53 Smith SM, Campbell NC, MacLeod U, et al. Factors contributing to the time taken to consult with symptoms of lung cancer: a cross-sectional study. Thorax 2009:64:523-31.

54 Slatore CG, Gould MK, Au DH, et al. Lung cancer stage at diagnosis: Individual associations in the prospective VITamins and lifestyle (VITAL) cohort. BMC Cancer 2011;11:1-7.

55 Starr LK, Osler M, Steding-Jessen M, et al. Socioeconomic position and surgery for early-stage non-small-cell lung cancer: a population-based study in Denmark. Lung Cancer 2013:79:262-9.

56 Stevens W, Stevens G, Kolbe J, et al. Ethnic differences in the management of lung cancer in New Zealand. J Thorac Oncol 2008:3:237-44.

57 Tweed C, Farley J, Horst JVD, et al. P169 Investigating the impact of social deprivation in lung cancer patients in North Glasgow. Thorax 2012:67(Suppl 2):A136.

58 Yorio JT, Xie Y, Yan J, et al. Lung cancer diagnostic and treatment intervals in the United States: a health care disparity? J Thorac Oncol 2009;4:1322-30.

59 NICE. Clinical Guideline 121 Lung Cancer: diagnosis and management. London: NICE, 2011

60 Jensen AR, Mainz J, Overgaard J. Impact of delay on diagnosis and treatment of primary lung cancer. Acta Oncologica 2002;41:147-52.

61 Egger M, Schneider M, Smith GD. Meta-analysis Spurious precision? Meta-analysis of observational studies. BMJ 1998;316:140-4.

62 Higgins J, Green S, eds. Cochrane handbook for systematic reviews of interventions (version 5.0.2). The Cochrane Collaboration, 2008.

63 Adams J, Ryan V, White M. How accurate are Townsend deprivation scores as predictors of self-reported health? A comparison with individual level data. J Public Health 2005;27:101-6.

64 Rubin G, Berendsen A, Crawford SM, et al. The expanding role of primary care in cancer control. Lancet Oncol 2015;16:1231-72. 